\title{
How can I do my postero-lateral thoracotomy without cutting any muscle of chest wall
}

\section{Comment je peux faire ma thoracotomie postéro-latérale sans sectionner au-} cun muscle de la cage thoracique

\author{
S. Rabiou ${ }^{1}$, F.Z. Ammor ${ }^{1}$, I. Issoufou ${ }^{1}$, L. Belliraj ${ }^{1}$, M. Lakranbi ${ }^{1}$, Y. Ouadnouni1 ${ }^{1,2}$, M. Smahi1 ${ }^{1,2}$ \\ 1: Service de Chirurgie Thoracique. CHU Hassan II de Fes. Maroc \\ 2: Faculté de Médecine et de Pharmacie. Université Sidi Mohamed Ben Abdellah -Fez. Maroc
}

\section{SUMMARY}

The common point for all thoracotomy is that it allows to approach the chest cavity. Several variants may exist depending on the nature of the gesture to be performed.

This may be the large posterolateral thoracotomy with complete muscular dissection of the big dorsal, the conservative thoracotomy with partial dissection of the big dorsal muscle or the hyper conservative thoracotomy is done without cutting any muscle of the chest wall. Indeed thoracotomy is a painful surgical approach and leaving very often the painful consequences for several years after the procedure. To minimize postoperative pain, the conservation of various muscle planes of the wall is a great alternative.

Thus the aim of this article is to describe step by step the technique of posterolateral thoracotomy without cutting any muscle of the chest wall which has its indications especially for small gesture like lung biopsy or during the exploration of the thoracic cavity.

KEYWORDS: Thorax, thoracotomy, postero-lateral thoracotomy

\section{RÉSUMÉ}

Le point commun de toute les thoracotomies est qu'elles permettent d'aborder la cavité thoracique. Plusieurs variantes existent en fonction de la nature du geste à réaliser.

Il peut s'agir de la grande thoracotomie postéro-latérale avec section musculaire complète du grand dorsale, la thoracotomie conservatrice avec section partielle du muscle grand dorsal ou encore la thoracotomie hyper conservatrice qui se fait sans sectionner aucun muscle de la paroi thoracique. En effet la thoracotomie est une voie d'abord très algique laissant souvent des séquelles douloureuses plusieurs années après le geste. Pour limiter la douleur postopératoire, la conservation des différents plans musculaires de la paroi est une excellente alternative.

Ainsi le but de cet article est de décrire pas à pas la technique de la thoracotomie postéro-latérale sans sectionner aucun muscle de la paroi thoracique qui trouve ses indications surtout pour des geste minime comme la biopsie pulmonaire ou lors de l'exploration de la cavité thoracique.

MOTS CLÉS: Thorax, thoracotomie, thoracotomie postéro-latérale 


\section{INTRODUCTION}

Le point commun de toute les thoracotomies est qu'elles permettent d'aborder la cavité thoracique. Plusieurs variantes existent en fonction de la nature du geste à réaliser. Il peut s'agir de la grande thoracotomie postéro-latérale avec section musculaire complète du grand dorsale, la thoracotomie conservatrice avec section partielle du muscle grand dorsal ou encore la thoracotomie hyper conservatrice qui se fait sans sectionner aucun muscle de la paroi thoracique.

En effet la thoracotomie est une voie d'abord très algique laissant souvent des séquelles douloureuses plusieurs années après le geste [1]. Pour limiter la douleur postopératoire, la conservation des différents plans musculaires de la paroi est une excellente alternative.

Ainsi le but de cet article est de décrire pas à pas la technique de la thoracotomie postéro-latérale sans sectionner aucun muscle de la paroi thoracique qui trouve ses indications surtout pour des geste minime comme la biopsie pulmonaire ou lors de l'exploration de la cavité thoracique.

\section{INSTALLATION}

Le patient est installé en décubitus latéral strict sur le côté sain. Le bras du côté a opéré est le plus souvent laissé tombant, protégé des appuis par des alèses. Un billot est placé sous l'omoplate opposé permettant ainsi d'ouvrir l'espace intercostal.

L'incision cutanée se fait selon une bissectrice de l'angle formé par la ligne des épineuses et le bord spinal de l'omoplate en passant à 2 travers de doigts en dessous de la pointe de celui-ci (Figure 1).

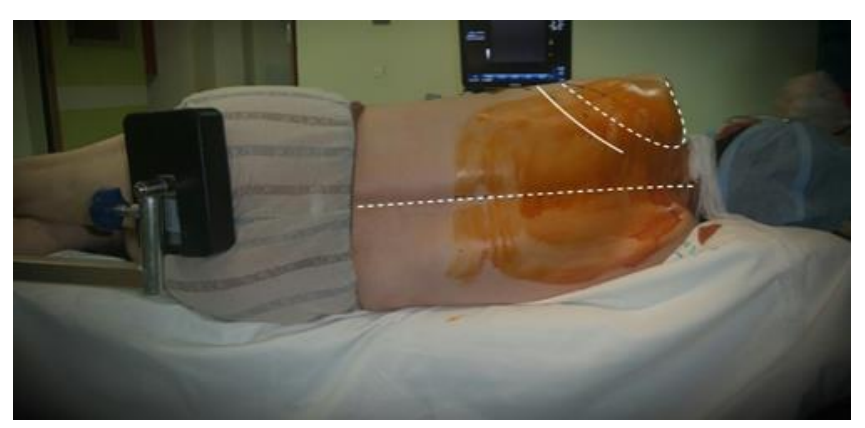

FIGURE 1. Installation.

\section{LES PLANS MUSCULAIRES}

\section{Le plan superficiel}

C'est le plan de muscle grand dorsal (ou latissimus dorsi) en avant et le trapèze en arrière. Dans la technique classique, le muscle grand dorsal est sectionné complètement avec ses pédicules vasculaires situés dans l'épaisseur du muscle.

Dans le cadre de la thoracotomie hyper conservatrice nous procédons à la dissection et au décollement de ces 2 gros muscles. Leur écartement avec les Farebef permet d'accéder au plan profond.

\section{Le plan profond}

Il comprend le muscle grand dentelé (ou serratus antérieur) en avant et en arrière, le rhomboïde ou (rhomboïdeus major). Ces 2 muscles sont unis par une aponévrose appelée aponévrose serratorhomboide.

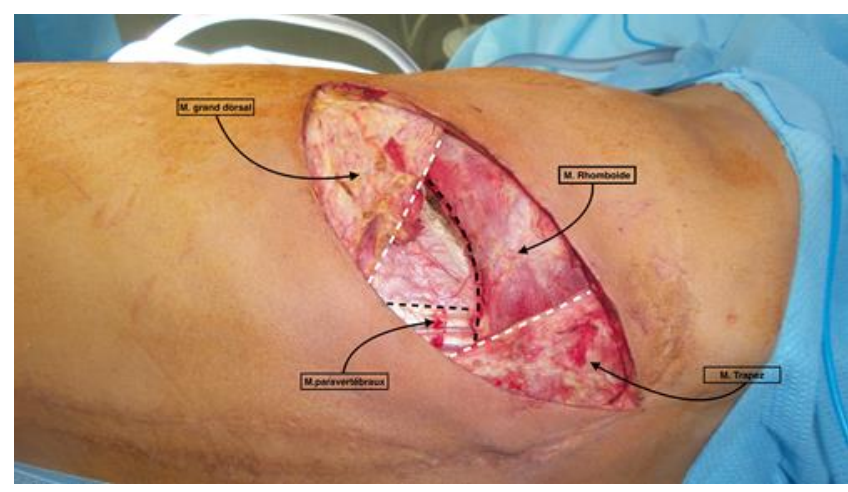

FIGURE 2. Le plan profond.

Dans la technique classique, le grand dentelé est sectionné au niveau de ses insertions basses. Nous préconisons sa désinsertion dans le cadre de cette thoracotomie sans section musculaire.

\section{L'OUVERTURE DE L'ESPACE INTERCOSTAL}

La dissection de ces différents plans musculaire permet d'exposer le plan costal, ainsi on peut glisser la main sous l'omoplate et compter les espaces intercostaux. La première côte perçue vers le haut correspond à la 2è côte (Figure $3 a$ ).

L'espace intercostal choisi est ouvert au raz de la côte inférieure, au bistouri électrique tout en respectant en arrière la masse musculaire paravertébrale.

L'ouverture de la cavité thoracique permet d'exclure le poumon ce qui facilite la mise en place de l'écarteur de Finochietto permettant de compléter l'ouverture de l'espace. On peut Ainsi explorer le thorax et réaliser des geste à minima comme une biopsie pulmonaire par exemple (Figure 3b). 


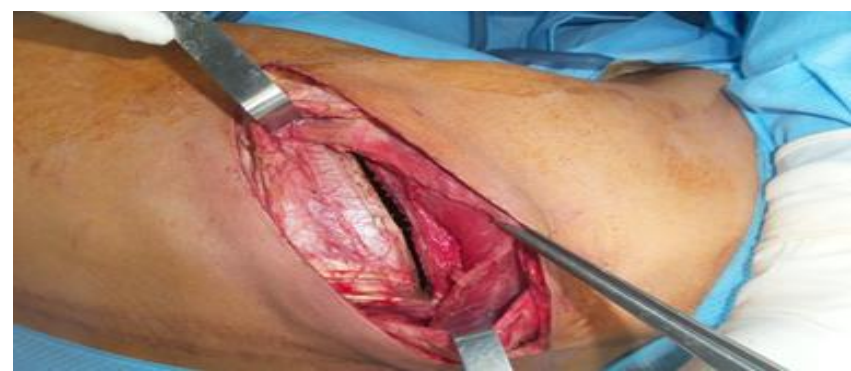

FIGURE 3a. L'ouverture de l'espace intercostale.

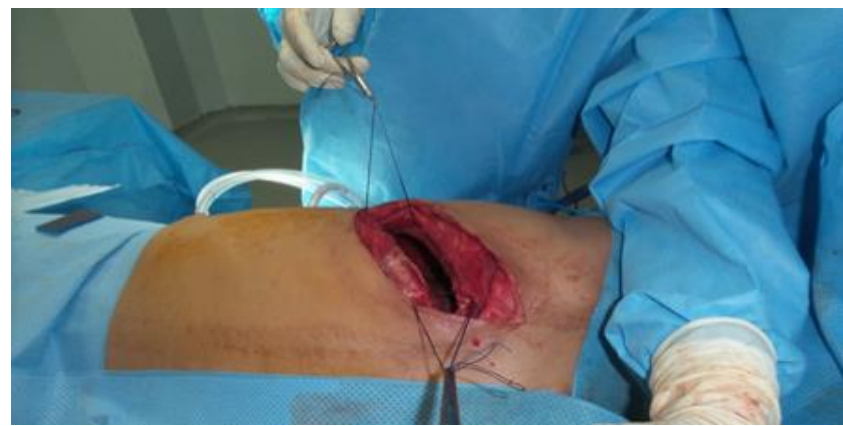

FIGURE 4a. Fermeture de la cage thoracique.

\section{LA FERMETURE}

Tout thorax ouvert doit être drainé.

Après la mise en place du, ou des drains, on enlève le billot permettant le rapprochement costal. 2 fils résorbables du Vicryl, $\mathrm{n}^{\circ} 2$ non serti, montées sur une aiguille de reverdin passant en trans-costal suffisent pour refermer l'espace intercostal (Figure 4a-b).

On procédé par la suite à la réinsertion des différents plans musculaire avant de fermer la peau.

\section{AVANTAGES}

Cette technique présente l'avantage de conserver le

\section{CONFLIT D'INTÉRÊTS}

Aucun.

\section{RÉFÉRENCES}

1. Akcali Y, Denir H, Tezcan B. The effect of standard posterolateral versus muscle-sparing thoracotomy on multiple parameters. Ann Thorac Surg 2003;76: 1050-4.

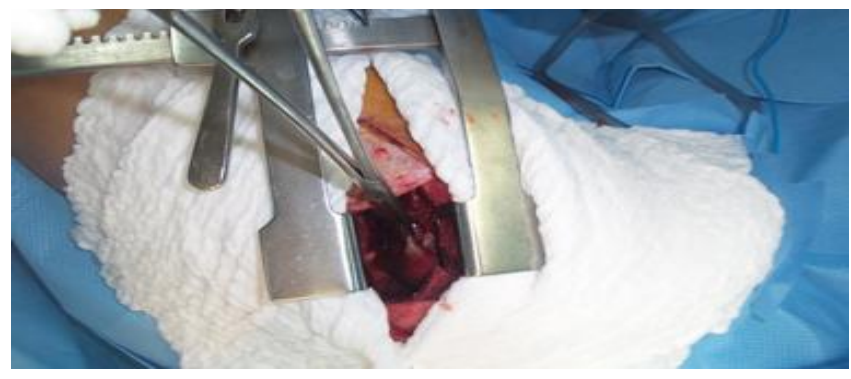

FIGURE 3b. L'ouverture de l'espace intercostale.

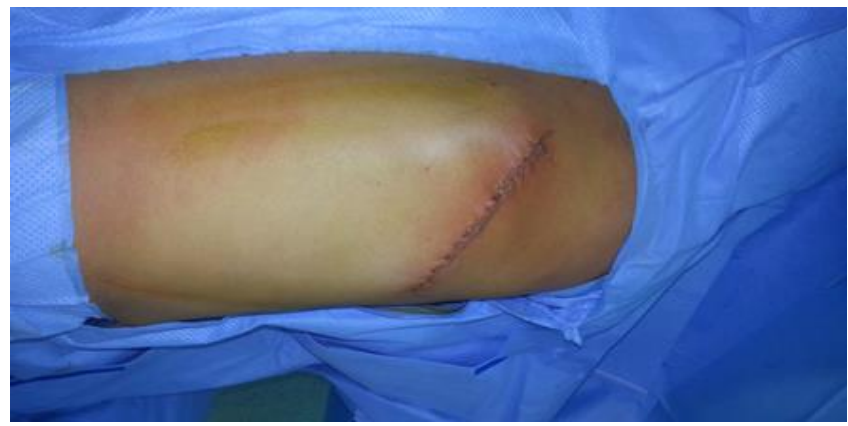

FIGURE 4b. Fermeture de la cage thoracique.

capital musculaire de la paroi thoracique qu'on pourra utiliser ultérieurement en cas de besoin pour des myoplasties ou thoracomyoplasties.

Sans compter le résultat esthétique, la conservation musculaire permet de limiter les douleurs postopératoires permettant au patient de bien faire sa kinésie thérapie respiratoire, étape essentielle pour la récupération de la fonctions respiratoire.

Il faut en plus noter que cette voie d'abord peut permettre de réaliser la plus part des gestes intrathoraciques comme la résection ou biopsie pulmonaire, la chirurgie pleurale et ou médiastinale notamment postérieur. 